\title{
Interactive comment on "Anthropogenic Influence on the Rhine water temperatures" by Alex Zavarsky and Lars Duester
}

\author{
Alex Zavarsky and Lars Duester \\ alexz@mailbox.org \\ Received and published: 13 January 2020
}

Interactive comment on "Anthropogenic Influence on the Rhine water temperatures "by Alex Zavarsky and Lars Duester

Anonymous Referee \#1Received and published: 26 November 2019

Introduction by the Authors: We would like to sincerely thank both reviewers for the comments and thoughts about our work and this manuscript. We think that the input significantly improved the manuscript. Based on the reviewers comments and by reviewing the code once again transposed numbers were found in the coding. By correcting the calculation method $A C C^{*} w$ provides the lowest RMSE and largest NCS in three out of four station. At the same time we were able to further decrease the RMSE 
for the $A C C^{*}$ w calculation method. The reasons for the $A C C^{*} w$ resulting in lower RMSE compared to ACC or w only, is now described in detail in the methods section. Overall, the results (correlations, RMSE, NCS, $\triangle$ RBTcalc) changed only so slightly, that the scientific conclusion and the key messages were not influenced. This also visible in the attached track changes version of the manuscript.

Interactive

\section{GENERAL COMMENTS}

The manuscript presents a study of short term and long term changes in river temperature and investigates the influence of natural and anthropogenic drivers of these changes which is interesting and generally within the scope of HESS. River temperatures at various monitoring locations along the river Rhine as well as industrial production and nuclear power plant activities are analyzed. The authors further develop a novel approach of calculating a catchment-wide average air temperature which is used in the linear regression relationship between air and river temperature. Overall, the scientific approach and the methods appear to be valid. However, there are some points which need further clarification:

(1) The relationships between river temperature and its drivers are investigated using multiple linear regressions separating the so-called Rhine base temperature (i.e. the river temperature without influences of air temperature and discharge) and air temperature and discharge influences on river temperature. More information on the multiple linear regressions for each location is required for the reader to be able to evaluate the robustness of this approach

Comment: The RMSE and the NCS information is provided for every measurement station. In addition, the data is now included in the supplement. We used the year 2001 as a test year and regressed Tw using Tc and Q just for this year. Then the 2001 regression coefficients were used to calculate a modelled Tw for the years 2000 to 2018. The RMSE and NCS show better results compared to the long term regression, which is sensible for a shorter regression period. The RMSE and NCS for each year

Printer-friendly version

Discussion paper 
from 2000 to 2018 follow the same pattern among the calculation methods. This means that the $A C C^{*} w$ method is always the best at three stations and the methods without time lag always show a larger RMSE than the ones with time lag (2) The computation of the catchment-wide average air temperature is based on the air temperature in each grid cell of the catchment area and the hydrological distance to the river temperature monitoring station assuming a constant flow speed. It would be interesting on what basis the constant flow speed has been derived and how the flow speed varies in space and time and what is the justification of combining a rather complex averaging method of air temperature with a constant flow speed. In order to show the benefits of this rather complex method, benchmarking with simple approaches (e.g. Catchment average air temperature in combination with constant lag time, as in Pohle et al., 2019) is suggested.

Comment: In our model, the flow speed does not vary in space and time. Generally, the flow speed in the shipping channel is between $1 \mathrm{~m} / \mathrm{s}$ and $2 \mathrm{~m} / \mathrm{s}$. This is supported by ADCP round robin tests (https://www.bafg.de/DE/05_Wissen/02_Veranst/2007/1009-07_bericht.pdf? blob=publicationFile) which showed a average flow speed of 1.2 $\mathrm{m} / \mathrm{s}$. Using the Koblenz data as reference we tried several flow speeds to minimize the RMSE of the model. We found a minimum of RMSE at $0.4 \mathrm{~m} / \mathrm{s}$. This is in the extended-range flow speeds. We expected a higher correlation at lower flow speeds than actually measured in the Rhine as we do not model standing water bodies. To us a flow speed with a magnitude difference would be questionable, but the one used is within reasonable limits.

(3) A data filter is used to compare river temperature and gross domestic product. It would be interesting how the filter parameters have been chosen and how sensitive the results are to different values of these filter parameters.

Printer-friendly version

Comment: We used a Butterworth band-pass filter instead of a running mean filter because the filter function of a butterworth is much easier to understand and it simply cuts all variations that are outside of the pass area. In this manuscript everything with

Discussion paper

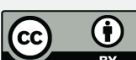


a periodicity of 20 years $(0.05 y-1)$ or longer is cut off. The reason is to eliminate long term trends, because the aim is to compare RBT to the GDP change. The lower limit is 0.9 years $(1.1 \mathrm{y}-1)$. Fast variations (faster than a year) of the RBT could influence the correlation vs a data-set (here the GDP) which is provided on a yearly basis. Therefore we smoothing is needed.

(4) As short-term and long-term changes of river temperature and its drivers are presented, it would be interesting to know if the data also show statistically significant trends and change points. The introduction section would benefit from more information and references to recently published literature. Also, the results need to be discussed with reference to related work and including appropriate reference to studies on river temperature. To that end, the authors are suggested to further familiarize with recently published studies on factors influencing river temperature (e.g. Garner et al., 2017; Lisi et al., 2015), river temperature modelling (e.g. Ketabchy et al., 2019; Wondzell et al., 2019; Zhu et al., 2019) as well as short-term and long-term changes in river temperature and its drivers (e.g. Basarin et al., 2016; Caldwell et al., 2015; Isaak et al., 2018; Pohle et al.,2019).The manuscript is overall well-written and structured. The results section includes many statements which would be better suited in the methods section. Further, I suggest adding a separate discussion section.

Comment: Thank you for pointing out additional literature. We added the rescaled adjusted partial sums to the manuscript. We checked trends of Tw and Ta at the four measurement stations and differences are visible. These differences are in accordance with our hypothesis that the progress of Tw at Worms, Koblenz and Mainz cannot be fully explained by the trend of Ta.

\section{SPECIFIC COMMENTS}

Page 1 - line 22 probably it is better to use "physical based" than "physical". Also, please check whether "deterministically" is the right term - probably it is referred to statistical models?

Printer-friendly version

Discussion paper 
Comment: Thank you, we changed the wording.

Page 2 - line 6/7 Is the statement by Markovic true for all rivers? (Their paper refers to Elbe \& Danube.)

Comment: We added the information that their study is based on Elbe and Danube data. As these two rivers are more or less comparable in size and catchment area to the Rhine, we think and also show that consistent results are given.

Page 2 - line 20 The equation is very specific and may be better suited in the "methods "part.

Comment: Thank you for the comment, but the fundamental idea of our hypothesis is to use the regression coefficients as explanation for changes in Tw. Therefore we need a simple linear $\mathrm{Ta} \rightarrow \mathrm{Tw}$ model. We want to present this idea and thought process in the Introduction. This is also done because we want to explain why we do not use hybrid, exponential models.

Page 2 - line 21 Suggestion to define coefficients already directly below the equation.

Comment: Thank you, we changed it.

Page 2 - line 25/26 Is this statement universal or only valid for the rivers studied in the cited papers - in that case please name these rivers.

Comment: We reorganized the references and specified to which subject the references addressed.

Page 3 - line 3 what is the original temporal resolution of the datasets? What were the procedures for quality control and have there been missing values?

Comment: The original resolution is $10 \mathrm{~min}$. We added a line to missing values and resolution in Sec. 2.1. The quality control is done by the sources. They initially verify the data-set. Additionally, the data-set was screened by us for suspicious features. 
Page 4 - Fig. 1 Please revise the map: make the river Rhine more visible, include monitoring stations and NPPs. Do the time lags refer to hydrological distance or to the grid? How have $0.733 \mathrm{~m} / \mathrm{s}$ been derived? How robust is this number - I would assume spatial \& temporal variability of flow speed.

Comment: We revised Fig. 1 which is now Fig. 2. The NPPs and measurement stations are now also included. We also describe in Sec. 2.7 how we obtained the flow speed and compare it to measured flow speeds.

Page 5 - tab. 2 How exactly have these values been derived?

Comment: We changed the table caption and added a few sentences in the "weighing coefficients" subsection, answering the question.

Page 7 - line 10 Sentence not needed.

Comment: We removed this sentence.

Page 7 - line 13-15 Suggest moving sentence to "methods" section.

Comment: These lines briefly explain Fig. 3. Hence, the authors think it should better remain in the Results section.

Page 8 - Fig. 3, tab. 3 Suggest adding 2nd figure column for air temperature. Merge figure and table (i.e. add slope values to the table). Please check robustness of number of digits of slopes, also state whether slopes are statistically significant.

Comment: We reduced the number of digits and added $R^{\wedge} 2$ values and a significance statement. We also added Ta in the figure and the RAPS index for trend analysis.

Page 8 - line 3 Which difference? It is stated that Ta warming rates are not really different. Comments: We added "from each other" to clarify this sentence.

Printer-friendly version

Page 9 - line 3/4 Please be more specific what is meant with "average European river"

Discussion paper

Comment: We removed this part. 
Page 9 - line 9/10 Move to "methods" section.

Comment: This is a brief reminder and explanation for Tab. 5. We prefer to keep it there.

Page $9 \& 10$ Combine tab. $4 \& 5$ and highlight the best model for each criterion \& location

Comment: We combined the tables and highlighted the best model.

Page 11 - tab. 6 what does "GW" stand for? Omit "the table shows"

Comment: Thank you, we replaced GW with " $\Delta \mathrm{Hi}[\mathrm{GW}]$ ". We removed "the table shows".

Page 11 - line 16 What is meant with "on average constant" - what time step does the average refer to?

Comment: The sentence was completely revised. P 16 Line 289.

Page 11 - line 25 Why has this particular company (BASF) been chosen?

Comment: It is close to the measurement station Worms and also provides significant heat input. We added this information to the manuscript.

Page 12 - line 2 Provide test statistics for significance or reword.

Comment: We omitted the word significant.

Page 14 - line 2 Linear models have also been applied elsewhere. However, it is unclear from this sentence how a linear relationship between air and river temperature implies universal applicability of the method presented in this paper. Furthermore, Morrill et al. found a better fit of non-linear models which might be even more pronounced outside of the tropics (i.e. conditions when air temperature, unlike river temperature, goes far below 0âŲ̧̊)

Printer-friendly version

Comment: The scope of this paper is not only finding a better (lower RMSE) way to 
model Tw, but to apply coefficients of a linear regression to better explain trend in Tw. more precise (etc.) models might be available, but most of them don't allow to distinct between anthropogenic, meteorological and hydrological impacts. If they allow this distinction, they are very labor-, time-, staff- and computing capacity intensive. This is not the case for the model proposed by us. Morrill et al. found suitable linear relationships between Ta and Tw for rivers around the world. This was a prerequisite for our analysis.

Page 15 - line 8 for reproducibility, please also name the data providers.

Comment: The data providers are mentioned in the methods section.

\section{TECHNICAL CORRECTIONS}

Page 1 - line 2/3 Sentence unclear - please revise.

Comment: Changed.

Page 1 - line 15 What does "their" refer to?

Comment: It refers to: energy intensive industries such as power plants, oil refineries, paper or steel mills. Changed to: "Its availability is a basic requirement for the facilitie's location (Förster and Lilliestam, 2010).

Page 2 - line 8 Please revise sentence structure.

Comment: We revised the sentence.

Page 2 - line 16 Please correct spelling to "assess"

Comment: Thanks we changed it.

Page 2 - line 24 Is the Markovic reference at the correct position of the sentence?

Comment: We changed the position.

Page 3 - tab. 1 Move table into methods section. 
Comment: It is in the methods section. The final formatting is applied by Copernicus.

Page 3 - line 13 Please correct to "European Centre for Medium-Range Weather Forecast".

Comment: Sorry, an awkward mistake. We changed it.

Page 3 - line 23 Hydrological distance between what? Noun missing.

Comment: Corrected.

Page 4 - line 12 Please consider moving reference to end of sentence.

Comment: We moved them.

Page 6 - line 3 "2019" instead of "20019"

Comment: We corrected it.

Page 7 - line 2 Better "reunification" as "unification" refers to 1871.

Comment: Typo, corrected.

Page 9 - line 9\&10 Nash-Sutcliffe ("e" missing").

Comment: We added an e.

Page 12 - Fig. 5 Y-Axis missing for Worms.

Comment: We added the axis.

Page 12 - line 3 Remove duplicate "by a".

Comment: Thanks, corrected.

Page 13 - line 2 Check spelling of "Mineralölraffinerie" and use the official name "Oberrhein" instead of "Karlsruhe".

Discussion paper

Comment: We changed that. 
Page 13 - line 2 Use Author (Year) citation format.

Comment: That's the formatting prescribed by Copernicus.

Page 14 - line 2 Remove given name from reference.

Comment: Changed.

Page 14 - line 10 Sentence unclear - "and" missing?

Comment: We corrected it.

Page 14 - line 15 Use Author (Year) citation format. Suggest to use "physical-based

Comment: That's the formatting prescribed by Copernicus.

\section{REFERENCES}

Basarin, B., Lukic, T., Pavic, D., \& Wilby, R. L. (2016). Trends and multi-annual variabil-ity of water temperatures in the river Danube, Serbia. Hydrological Processes, 30(18),3315-3329. https://doi.org/10.1002/hyp.10863

Caldwell, P., Segura, C., Laird, S. G., Sun, G., McNulty, S. G., Sandercock, M., et al. (2015). Short-term stream water temperature observations permit rapid assessment of potential climate change impacts. Hydrological Processes, 29, 21962211.https://doi.org/10.1002/hyp.10358

Garner, G., Malcolm, I. A., Sadler, J. P., \& Hannah, D. M. (2017).The role of riparian vegetation density, channel orientation and water velocity in determining river temperature dynamics. Journal of Hydrology, 553(September), 471485.https://doi.org/10.1016/j.jhydrol.2017.03.024

Isaak, D. J., Luce, C. H., Horan, D. L., Chandler, G. L., Wollrab, S. P., \& Nagel, D. E.(2018). Global Warming of Salmon and Trout Rivers in the Northwestern U.S.: Road to Ruin or Path Through Purgatory? Transactions of the American Fisheries Society. https://doi.org/10.1002/tafs.10059 
Jackson, F. L., Fryer, R. J., Hannah, D. M., Millar, C. P., \& Malcolm, I. A.(2018).A spatiotemporal statistical model of maximum daily river temperatures to inform the management of Scotland's Atlantic salmon rivers under climate change. Science of the Total Environment, 612(January), 15431558.https://doi.org/10.1016/j.scitotenv.2017.09.010

Ketabchy, M., Sample, D. J., Wynn-Thompson, T., \& Yazdi, M. N. (2019).C6 Simulation of watershed-scale practices for mitigating stream thermal pollution due to urbanization. Science of the Total Environment, 671, 215231.https://doi.org/10.1016/j.scitotenv.2019.03.248

Lisi, P. J., Schindler, D. E., Cline, T. J., Scheuerell, M. D., \& Walsh,P. B. (2015).Watershed geomorphology and snowmelt control stream thermal sensitivity to air temperature. Geophysical Research Letters, 42(9), 1-9.https://doi.org/10.1002/2015GL064083

Piccolroaz, S., Calamita, E., Majone, B., Gallice, A., Siviglia, A., \& Toffolon, M. (2016).Prediction of river water temperature: a comparison between a new family of hybrid models and statistical approaches. Hydrological Processes, 30(21), 39013917.https://doi.org/10.1002/hyp.10

Comment:Real good paper that shows the difference between Lake fed, regulated, snow fed and low land rivers.

Pohle, I., Helliwell, R., Aube, C., Gibbs, S., Spencer, M., \& Spezia, L.(2019).Citizen science evidence from the past century shows that Scottish rivers are warming. Science of the Total Environment, 659, 53-65.https://doi.org/10.1016/j.scitotenv.2018.12.325

Wondzell, S. M., Diabat, M., \& Haggerty, R. (2019). What Matters Most: Are Future Stream Temperatures More Sensitive to Changing Air Temperatures, Discharge, or Riparian Vegetation? Journal of the American Water Resources Association, 55(1),116132. https://doi.org/10.1111/1752-1688.12707

Zhu, S., Heddam, S., Nyarko, E. K., Hadzima-Nyarko, M., Piccolroaz, S.,

Printer-friendly version

Discussion paper
Interactive

comment

\section{C11}


\& Wu, S.(2019). Modeling daily water temperature for rivers: comparison between adaptiveneuro-fuzzy inference systems and artificial neural networks models. EnvironmentalScience and Pollution Research, 26(1), 402-420. https://doi.org/10.1007/s11356-018-3650-2

Hoef, J. M. V., Peterson, E., \& Theobald, D. (2006). Spatial statistical models that use flow and stream distance. Environmental and Ecological Statistics, 13(4), 449-464. doi:10.1007/s10651-006-0022-8 âǍČ Reviewer 2

Interactive comment on "Anthropogenic Influence on the Rhine water temperatures" by Alex Zavarsky and Lars Duester

In this study, the authors analyze the effects of Nuclear Power Plants on river water temperature of the Rhine. The authors propose a multiple linear regression model where river water temperature is simulated based on air temperature and streamflow as predictor variables. Air temperature is evaluated through an averaging procedure that accounts for the geomorphology of the hydrological catchment. The intercept of the multiple linear regression models is used as a proxy for the anthropogenic impact on river water temperature and is compared to the time series of GDP and heat input from NPPs. The presentation of the methodological approach and of the results should be improved, both in terms of clarity and quality. In my opinion the robustness of some methodological aspects is weak (e.g., the use of a constant flow velocity, the interpretation of the multiple linear regression intercept as "indicator for industrial heat input") and the discussion of the results should be expanded and deepened. The literature review on modeling of river water temperature and assessment of anthropogenic impacts should be updated and the grammar and syntax of the manuscript should be checked carefully. Please, find below some specific comments.

Specific comments

Introduction:

Printer-friendly version

Discussion paper
Interactive

comment 
The literature review on modeling of river water temperature should be expanded and updated including the most recent studies in this field. Besides "classical" deterministic and statistical models, there is a wide range of models based on machine learning techniques or hybrid physically-based/statistical approaches (e.g., Sahoo et al., 2009; Toffolon and Piccolroaz, 2015; Sohrabi et al., 2017), which have been emerging in the last years. Despite it is not recent, I suggest giving a look to the review paper by Benyahya et al. (2007), which provides a good overview of deterministic and statistical models used in the field of river water temperature prediction. Another useful and more recent paper is that by Gallice et al. (2015).

Comment: Another thorough literature search was undertaken and we added among other references, the references proposed by both reviewers. The overview of water temperature models was extended in the introduction.

In addition, the authors should refer also to existing literature on the assessment of anthropogenic impact on river water temperature (e.g., Cai et al., 2018; Gaudard et al., 2018; Raman Vinna et al., 2018,just to cite some recent papers).

Comment: The publications were cross-checked. The input was included in the revision of the manuscript.

In general, I believe that the paragraph from P1, line 19 to P2, line 8, should be thoroughly restructured and revised, and the authors should be more precise throughout the text (e.g., at P1, line 22: I believe that the authors intend deterministic and statistical models here; at P2, lines 21-23, the sentence is unclear; at P2, lines 25-26, the comment is superfluous since in a multiple linear regression, such as the one used by the authors, these components are obviously neglected). P2, lines 7-8: I would rephrase this sentence in more general terms, because the amount of variance in river water temperature explained by air temperature and streamflow are strongly dependent on the case study (hydrological regime, season, etc.).

Printer-friendly version

Comment: Thank you for the comments. We revised the whole introduction. The 
changes we made can be seen in the track changes version. P2 lines 25-26: We know that our model does exclude ground heat flux and friction. If the parameters are important they would appear most likely and unfortunately in the regression coefficient a1. However, a1 is the basis of our analysis which should display the anthropogenic heat input We just want to say that we think these heaf fluxes are neglible and do not interfere with our anthropogenic heat input. In this regard, the authors should expand the analysis of parameters a2 and a3 of their regression model. The second half of the Introduction (from P2, line 16) should be moved to the methods section and should be improved, as in its current form it does not clearly describe how the authors set up their analysis, especially concerning the definition and use of RBT as an "indicator for industrial heat input" and the time resolution of the data used in the multiple linear regression analysis. Figure 1 This figure should be updated with the location of the monitoring station and of the NPPs. The main course of the Rhine should also be indicated. Comment: We changed Figure 1. In the introduction we give just a basis overview of our idea which is closely linked to the linear regression model. We moved some parts to the methods section. The detailed calculations are described in the methods section.

Section 2.1. I agree on the comment about accuracy and precision, however I wonder if the measurements are affected by instrumental drift and, in case, if the dataset has been corrected accordingly.

Comment: The data was verified by the data provider(e.g., by recurrent validation measurments, recalibration if needed or cross-validation). The data-set was screened for suspicious features. We stated this in the manuscript.

P3, line 9: this sentence is unclear. In general, I agree that water temperature is rather homogeneous at a river section if it has a compact geometry, while it may be non-uniform if the geometry is complex.

Comment: We know that the measured water temperature, especially in complex river

Printer-friendly version

Discussion paper
Interactive comment 
geometries, is an on-spot in-situ temperature and could be different from a crosssection average Tw. However, a method benefit of this analysis is that only the water temperature differences are needed. If the measured Tw changes and the crosssection Tw does, accordingly.

Section 2.2. Here the authors used a constant flow speed to evaluate the flow time required to travel from a cell of the catchment to the catchment outlet. The authors should clarify how they selected this flow speed and if it is reasonable to assume a constant value (was this velocity the same for the four outlets?). I wonder about the methodological robustness of the approach proposed by the authors since they applied the same flow velocity to all cells pertaining to the catchment, thus both to hillslope and river network cells. In this regard, I also do not fully agree on the sentence at P5, lines 21-22 since before reaching the channel network, rainfall may follow different paths (infiltration,C3runoff, etc.), thus exchanging heat with the surrounding environment and decreasing its correlation to Ta.

Comment: PIs. cf. GENERAL COMMENTS (2) to reviewer 1.

P3, line 20:

Comment: We changed the wording.

P4, line 1

Comment: We changed the wording

Section 2.3 The authors state that parameter a1 (the intercept) summarizes all effects that are not directly ascribable to Ta and $Q$, which "are mostly from anthropogenic sources". Personally, I do not agree that, in general, the value of a1 can be unequivocally related to anthropogenic factors.

Printer-friendly version

Comment: Of course there is no proven, but this the hypothesis. We are able to strongly support this hypothesis by comparing changes in anthropogenic heat input (nuclear power plants) and short term economic changes to a1 and draw a consistent picture in 
the manuscript.

The authors should support this statement referring to previous literature on the topic. In this regard, a useful reading is Isaak et al (2011), where also the multiplicative interaction term has been included in the multiple linear regression model.

Comment: We reviewed all citations, thank you for the hints. If applicable we changed the manuscript. Especially, the different methods for modelling Tw are described now more detailed in the introduction.

Variables $x 0, y 0$, and in eq 2 are not defined. Table 2 (and corresponding description in the main text): the authors should provide details on why they assumed a linearly deceasing weighting factor instead of other weighting functions.

Comment: We added an explanation of $\mathrm{x}, \mathrm{y}$. We revised our model and use now $\mathrm{ACC}^{*} \mathrm{~W}$ as weighting factor. The reason for a linear decrease cannot be answered within this manuscript and more research is needed.

While the weighting factors decreases with $\Delta t$, I expect that Tw is no more correlated to Ta after some time. The authors obtain the best results using the "Time lag" model instead of the "Time lag + weight" model, saying that the furthest and oldest Ta influences on Tw are still carried as information in the water mass (P9, lines 4-5). In my opinion, the real reason is that without assuming a deceasing weighting factor the authors increase the dependence of current river water temperature on previous conditions, thus implicitly accounting for the thermal inertia of the river. This is an important aspect controlling river water temperature, which is not explicitly included in the model proposed by the authors and that can be accounted for e.g., through autocorrelation terms (e.g., Caissie et al., 2001; Toffolon and Piccolroaz, 2015).

Comment: We think that the reason for using a weighting factor decreasing is a) to put less weight on the large amount of grid-points with less ACC and b) to put less weight on temperatures with a large $\Delta t$. Autocorrelation is an option but we decided not use it

Printer-friendly version

Discussion paper
Interactive

comment 
for this model.

Control scenarios I would use a different word than "scenarios" here, since these are not scenarios but different approaches to calculate Tc .

Comment: Changed.

Section 2.4 The authors should explain how they calculated the heat input by NPP to the Rhine. The section should be expanded, and the sentences harmonized to make the reading more fluid (too short sentences).

Comment: We moved the explanation of the NPP heat input to the methods section and revised it.

Figure 3 and Table 3 Figure 3 would benefit from the inclusion of the air temperature time series with the corresponding linear trends. This would be useful for better understanding the correlation between river water temperature and air temperature fluctuations, which are filtered out when using linear trends. In this regard, it would be useful to add the Pearson correlation coefficient between these two variables in Table 3.

Comment: We added air temperature to the figure. We also added the RAPS index to make trends more visible.

At P8, lines 12-15 it would be useful to compare the trends found by the authors with those of more recent studies.

Comment: We removed this section. The focus of the paper is on providing reasons for the heterogeneous Tw trends in the Rhine river, an urgent matter in regulative river heat evaluation in times of climate change.

Tables 4 and 5 Why did the authors use the "Time lag weight" approach for all other results instead of the "Time lag" approach, which performed the best? It should be clearly indicated if the RMSE and NSC refer to daily or annual values.

Printer-friendly version

Comment: As mentioned before (first page of this document), the data was reanalyzed. 
As a consequence the tables and parts of the results were revised. The scientific conclusion was not changed.

Section 3.3 It is unclear how the authors evaluated RBT over time. Did I correctly understand that they applied the multiple linear regression model for overlapping twoyear time windows shifted by one month? What was the rationale of assuming two-year time windows instead of longer periods? Are the results affected by the length of the time window used for this analysis?

Comment: Longer time windows would decrease the temporal resolution of the regression. A shorter time window increases the influence by other linear dependent influences. The two years were chosen to address two full annual cycles. If a year was extraordinary concerning air temperature or discharge, a two year cycle would not be prone to such events.

P10, line 2 these sentences are qualitative, and not sufficiently supported by the results.

Comment: We changed the wording. We add that we cannot meaningfully interpret the absolute value RBT.

$\mathrm{P} 11$, line 4 : these sentences are qualitative, and not sufficiently supported by the results.

Comment: The similar trends are supported by the analysis comparing calculated $\Delta$ RBT with measured $\Delta$ RBT.

The comment on the effect of alpine lakes is not well connected to the rest of the paragraph and should be expanded with some more detailed discussion.

Comment: We just hypothesize why Basel has such an alternating RBT. However, the RBT does not show a long term trend over the whole dataset. Finding the reason is not in the scope of this paper. 
Eq 10 is dimensionally not consistent.

Comment: Thank you, we missed the density. Changed.

How did the authors select the periods in Table 6?

Comment: The start of the period is the time of the maximum heat input by NPPs at the respective station. We added this information to the text.

The authors could do the same calculation in continuous, for the entire period when the data are available (e.g., using the same two-year time windows as before).

Comment: This would be a good idea. However, tha aim was to use a time windows with the largest signal to noise ratio. Therefore we picked the largest $\Delta \mathrm{HI}$ to avoid influences by short term trends.

P11, line 16: what is the BASF company? This should be explained.

Comment: We added two sentences to explain the BASF.

Why RBT in Figures 4 and 5 are different? How sensitive are the results of the correlation analysis to the filtering of the data?

Comment: Figure 5 has filtered RBT, cf. comment on the reviewer 1 on page 2

How the filtering parameters have been chosen and why 480 days has been used to shift the GDP-change time series? This number seems quite arbitrary.

Comment: It was shifted to ensure a visual match between the two data-sets (GDP and RBT). The shift can be explained by lagging and leading economic factors. This is explained in the manuscript. Mathematically the 480 days shift does not yield the largest positive correlation.

Appendices could be moved to the main text. In particular, the sentences in Appendix B should be revised because they have some syntax errors and typos. Figures A1 andA2 are inverted and the caption is the same. The analysis of parametersa2anda3should

Printer-friendly version

Discussion paper 
be deepened and moved to the main text.

Comment: We move the biggest part of the appendix into the main text, as advised.

\section{Technical corrections}

P1, line 13: "but an" $\rightarrow$ "but is an". Is "means of production" an appropriate term in thiscontext?

Comment: Thank you for the hint. We think means of production is appropriate.

$\mathrm{P} 2$, line 3 and following lines: the use of "Ta $\rightarrow \mathrm{Tw}$ " is informal and should be modified.

Comment: Thank you for your comment but we would like to keep it that way.

P2, line 8: "hydro-logical" -> "hydrological"

Comment: We changed it.

P2, lines 8-9: a reference is needed here.

Comment: This part has been moved and we added a reference in this sentence.

$\mathrm{P2}$, line 16: is "revise" the most appropriate term here?

Comment: You are right. We use "test" now.

P2, line 20: "almost ideal" -> "ideal", "interesting", "meaningful"

Comment: Thank you, we changed it.

P4, line 13: "followed, by" -> "followed by". Please, thoroughly revise the punctuationthroughout the article (use of commas, missing close-brackets, etc).

Comment: We completely revised this part. The sentence is now rewritten.

P5, line 17: "ptovided" -> "provided"

Discussion paper

Comment: We changed it. 
P6, line 1: I would say that authors present fourTccalculations, not two.

Comment: We revised this part completely.

P6, line 18: "heat input by NPPsto the Rhine" -> "heat input by NPP to the Rhine"

Comment: We changed it.

P8, line 5: "(0.0350âŮęCy-1)" -> "(0.0489âŮęCy-1)"

Comment: We completely revised this table.

P10, line 15: "over the a time period" -> "over a time period"

Comment: Thank you, we changed it.

P11, line 1: "shorter timer scale but do not seem,to our" $\rightarrow$ "shorter time scale but doC6 not seem, to our"

Comment: Thank you, we changed it.

$\mathrm{P} 11$, line 14: "A a discontinuity" -> "A discontinuity"

Comment: Changed. P11, line 19: "by a by a" -> "by a"

Comment: Changed.

References Benyahya L., Caissie D., St.Hilaire A., Ouarda T.B.M.J., Bobe B. 2007. A review of statistical water temperature models. Canadian Water Resources Journal 32: 179-192 Cai H., Piccolroaz S., Huang J., et al.2018. Quantifying the impact of the Three Gorges Dam on the thermal dynamics of the Yangtze River. Environ ResLett.;13(2018):054016. Caissie D., El Jabi N., Satish M.G. 2001. Modelling of maximum daily water temperatures in a small stream using air temperature. Journal of Hydrology 251: 14-28. Caissie D., Satish M.G., El-Jabi N. 2005. Predicting river water temperatures using the equilibrium temperature concept with application on Miramichi River catchments (NewBrunswick, Canada) Hydrol. Process. 19 2137-59 Gaudard, A, Weber, C, Alexander, TJ, Hunziker, S, Schmid, M. 2018. Impacts of using lakes 
and rivers for extraction and disposal of heat. WIREs Water. 5:e1295. Gallice A., Schaefli B., Lehning M., Parlange M.B., and Huwald H. 2015. Stream temperature prediction in ungauged basins: review of recent approaches and description of a new physics-derived statistical model. Hydrol Earth Syst Sci 19:3727-3753 Isaak D.J., Luce C.H., Rieman B.E., Nagel D.E., Peterson E.E., Horan D.L., Parkes S., Chandler, G.L. 2010. Effects of climate change and wildfire on stream temperature sand salmonid thermal habitat in a mountain river network. Ecol. Appl. 20, 1350-1371. Isaak D.J., Wollrab S., Horan, D., Chandler, G. 2011. Climate change effects on stream and river temperatures across the northwest U.S. from 1980-2009 and implications forsalmonid fishes. Climatic Change. 113: 499-524 Sahoo G.B., Schladow S.G., and Reuter J.E. 2009. Forecasting stream water temperature using regression analysis, artificial neural network, and chaotic non-linear dynamicmodels. J. Hydrol. 378 325-42 Sohrabi M.M., Benjankar R., Tonina D., Wenger S.J., and Isaak D.J. 2017. Estimation of daily stream water temperatures with a Bayesian regression approach. Hydrol.Process. 31, 1719-1733 Toffolon M., and Piccolroaz S. 2015. A hybrid model for river water temperature as a function of air temperature and discharge. Environmental Research Letters 10:114011 van Vliet M.T.H., Ludwig F., Zwolsman J.J.G., Weedon G.P., Kabat P. 2011. Global river temperatures and sensitivity to atmospheric warming and changes in river flow. WaterResour. Res. 2011, 47 Raman Vinna L., Wüest A., Zappa M., Fink G., Bouffard, D. 2018. Tributaries affect thethermal response of lakes to climate change, Hydrol. Earth Syst. Sci., 22, 31-51

Interactive comment on Hydrol. Earth Syst. Sci. Discuss., https://doi.org/10.5194/hess-2019518, 2019. 